\title{
Imaging Properties of Multimode Photonic Crystal Waveguides and Waveguide Arrays
}

\author{
Daniele Modotto, Member, IEEE, Matteo Conforti, Andrea Locatelli, and Costantino De Angelis, Member, IEEE
}

\begin{abstract}
Light propagation in multimode photonic crystal waveguides and in arrays of single-mode photonic crystal waveguides is analyzed. The dispersion relations of the modes travelling along these devices explain the numerically observed formation of single and multiple copies of the input beam. As the analogous devices constructed in standard planar geometry, the proposed photonic crystal devices can be employed as filters and demultiplexers. The use of photonic crystal structures, where the light is truly confined by the stop-band, adds the valuable benefit of a dramatic reduction of the overall geometrical dimensions.
\end{abstract}

Index Terms-Couplers, diffraction, finite element method, integrated optics, multimode interference, multimode waveguides, photonic crystal, waveguide arrays.

\section{INTRODUCTION}

$\mathbf{P}$ HOTONIC crystal (PC) based devices are paving the way for a new generation of integrated optical circuits [1],[2]. In fact PC components, by taking advantage of the light stop-band, allow to shrink the dimensions of the conventional optical chips [3]. A 2-D periodic structure, like a lattice of dielectric rods immersed in a low refractive index material or a lattice of holes in a high refractive index substrate, can be engineered in order to obtain an in-plane stop-band for the propagation of light. By reducing or increasing the radius of the rods or of the holes (i.e. by introducing defects) a waveguide working over a span of wavelengths can be fabricated [4].

Obviously a 2-D structure cannot be used in practice: it is necessary to achieve confinement in the vertical direction, as well [4]. The most intuitive solution is to sandwich the 2-D structure between an upper and a lower cladding whose refractive indices are carefully chosen [5],[6]. For example a periodically drilled dielectric membrane could be suspended in air [7] or a low refractive index region with high refractive index posts could be grown on a proper substrate [5]. It has been noticed that even if the refractive index difference between the background material hosting the rods and the cladding is small, the 3-D PC waveguide may suffer from negligible losses and moreover the design parameters (rod radius and lattice pitch) do not differ appreciably from those obtained by a straightforward 2-D analysis [5],[8].

As with conventional ridge optical waveguides, a great effort has been devoted to the project of single-mode waveguides. This design goal is particularly challenging for PC waveguides constructed by perturbing a lattice of rods: it is well known that by increasing the radius of the rods arranged along a

The authors are with the Dipartimento di Elettronica per l'Automazione, Università di Brescia, via Branze 38, Brescia 25123, Italy. straight line, the resulting waveguide could support more than one mode.

Nevertheless conventional multimode waveguides can be exploited in a large variety of coupling and splitting devices [9],[10]. In fact, when an optical field is properly launched in a multimode waveguide, a certain set of modes is excited and since the modes travel with different phase velocities, copies or multiple images of the initial field form at particular distances [11],[12],[13]. Recently PC multimode waveguides have been proposed as demultiplexers [14] and 3-dB power splitters [15]. Useful rules for the design of practical and compact splitting and routing devices are available for conventional waveguides [13] and in this communication we prove these rules to be helpful even for PC multimode waveguides.

A thoroughly studied PC device, where multimode interference takes place, is the directional coupler [16],[17]; its behavior can be explained also resorting to the coupled mode theory (CMT) which gives a clear picture of the flow of energy between the waveguides. PC based directional couplers have gained a renewed interest because, by controlling the spacing between the waveguides, the coupling coefficient between adjacent waveguides can be engineered both in amplitude and sign [18],[19],[20].

By increasing the number of coupled waveguides an array is obtained [21],[22], [23],[24] and, as in directional couplers, the field evolution is governed by a set of coupled equations where the coupling coefficient plays a crucial role. We have verified that a similar set of equations describes light propagation along an array of PC waveguides [19],[20]. We observe that a PC waveguide array, considered as one single system, supports several supermodes and the numerical study of the field propagation permits to choose the device length when splitting or filtering functionalities are required.

The properties of single-mode and multimode PC waveguides are introduced in Section II whereas the self-imaging is discussed through examples in Section III. Starting from an outline of the behavior of PC couplers in Section IV, the arrays of PC waveguides are discussed in Section $\mathrm{V}$ where their potential as filters and demultiplexers is demonstrated.

\section{Single-MOdE AND MULTIMOdE PHOTONIC CRYSTAL WAVEGUIDES}

We consider 2-D guiding structures obtained by perturbing a square lattice of dielectric rods embedded in an oxide background. A good choice in order to obtain a single mode waveguide is to decrease the radius of the rods arranged along a row and in the following examples we decided to completely remove these rods. 
The dispersion relation can be calculated via the plane wave expansion method [25]; the elementary cell, which includes the perturbed region, has length along the propagation direction $z$ equal to the lattice constant $a$ and it is wide enough along the transverse direction $x$ [1],[25].

We analyse a bulk lattice of semiconductor ( $\mathrm{GaAs}$ ) rods having refractive index 3.34 embedded in oxide $\left(\mathrm{Al}_{2} \mathrm{O}_{3}\right)$ whose refractive index is 1.5 [5]; the rod radius is $r=117$ $\mathrm{nm}$ and the rod-rod pitch is $a=390 \mathrm{~nm}$. The projected band structure (along $z$ ) of the PC waveguide obtained by removing a row of rods is depicted in Fig.1. The dark-shaded regions indicate the continuum of dispersion curves of the extended modes of the unperturbed lattice, the white region is the stopband (ranging from $a / \lambda=0.234$ to $a / \lambda=0.266$ ) and the thick solid line is the dispersion relation of the only TM mode that can propagate in the waveguide. We emphasize that the normalized wavevector ( $\beta a / 2 \pi$, where $\beta$ is the wavevector) is an increasing function of the normalized frequency $(a / \lambda)$. The horizontal narrow line indicates the design wavelength $\lambda=1550 \mathrm{~nm}(a / \lambda=0.2516)$. The corresponding $2-\mathrm{D}$ mode profile is shown in Fig.2: most of the energy is confined in the perturbed region (as happens in the core of conventional slab waveguides) but the field tails outside the perturbed regions have an oscillating behavior which has no counterpart in 3layer slab waveguides.

The multimode PC device can be obtained by removing the rods along a few neighbouring rows. The input field is injected directly at the waveguide input facet or by means of a singlerow (and single-mode) waveguide as illustrated in the sketch of Fig.3.

As an example we consider a device obtained by removing seventeen rows from the above described lattice $(a=390 \mathrm{~nm}$, $r=117 \mathrm{~nm}$ ). The projected band structure is displayed in Fig.4: the white region is the stop-band and the solid black lines are the dispersion relations for the fourteen TM guided modes. We observe that at the reference wavelength $\lambda=1550$ $\mathrm{nm}$ only thirteen modes are supported.

The 2-D modes are shown in Fig.5; as in standard slab waveguides the modes exhibit "sin-like" shapes in the region where most of the energy is concentrated [13],[26]. The studied PC device (where the rods are completely removed) exhibits a very strong confinement in the lateral direction $x$ and the region where the field is concentrated has a uniform refractive index: it is thus not surprising that the guided modes resemble those supported by slab waveguides where the confinement is guaranteed by a strong refractive index contrast or by metallic surfaces.

\section{SElf-imaging IN MUltimode PHOTONIC CRYSTAL WAVEGUIDES}

In planar waveguides supporting several modes, the formation of single or multiple copies of the input field can be observed. These copies are formed along the propagation direction at precise distances that can be related to the beat length $L_{\pi}$ between the two lowest order modes of the waveguide [12],[13]:

$$
L_{\pi}=\frac{\pi}{\beta_{0}-\beta_{1}}
$$

where $\beta_{0}, \beta_{1}$ are the propagation constants of the fundamental and second mode, respectively. The presence of high order modes is necessary to observe the formation of multiple images but, since it is extremely important also the difference among the propagation constants of the modes [12],[13], the onset of self-imaging phenomena in a PC multimode waveguide is not a trivial result.

Due to the complexity of the dielectric structure under investigation, we analyse the electromagnetic field propagation along the PC multimode waveguide by numerically solving Maxwell's equation (in the vector wave equation form) through a Time Domain Vector Finite Element Method (TDVFEM) [27],[28],[29],[30].

TDVFEM offers some important advantages over the standard and widely used Finite Difference Time Domain Method (FDTD). The most evident one is the use of unstructured grids which offer superior versatility in modelling complex geometries and permit the number of degrees of freedom to be greatly reduced to achieve the same accuracy. We employ the so called "vector" or "edge" elements of lowest order [31], [32] which have degrees of freedom along the edges of the grid. These elements enforce tangential continuity of the fields but allow for jump discontinuity in the normal component, which is a requirement for accurate modelling of fields in inhomogeneous domains. Moreover this characteristic eliminates the problem of spurious solutions typical of the classic nodal elements [33]. Crank-Nicolson method is employed to advance fields in time. This method is second-order accurate, energy-conserving and unconditionally stable. This last feature allows one to fix time step and mesh granularity independently, in contrast with FDTD where one has to respect the stability condition. The main drawback of TDVFEM with respect to FDTD is that it requires a sparse linear system to be solved at every time step. However the system matrix is very sparse (around $5 \times N$ nonzero elements, being $N$ the number of degrees of freedom) and the system can be efficiently solved by the use of preconditioned conjugate gradient (typically less than ten iterations are required to obtain the desired accuracy). Thanks to these good properties, TDVFEM can solve very large problems with a reasonable time and memory consumption and can overcome the severe limitations imposed by the staircase approximation and the stability condition of FDTD.

By using TDVFEM we excite the designed PC waveguide (whose dispersion relations are shown in Fig.4) with the fundamental mode of a single row waveguide positioned in the middle of the multimode device (see Fig.3). The timeaveraged intensity depicted in Fig. 6 shows the formation of a clear copy of the input beam at the distance $z=43.5 \mu \mathrm{m}$.

In a planar waveguide and for a symmetric input, the first copy is expected at a distance $3 L_{\pi} / 4$ [13]. From the calculated dispersion relations of the PC multimode waveguide (Fig.4) we obtain $\beta_{0}=0.3763 \times 2 \pi / a, \beta_{1}=0.3731 \times 2 \pi / a$ and thus $3 L_{\pi} / 4=45.7 \mu \mathrm{m}$ which coincides fairly well with the result shown by the simulation in Fig.6. We conclude that since the field is almost completely confined in the uniform oxide layer (due to the presence of the bandgap), the relation reported in [9],[13] still holds to some extent. 
As reported for conventional integrated optics multimode devices, the formation of multiple images takes place, as well. A double copy of the input image can be observed around $z=$ $22 \mu \mathrm{m}$ (to be compared with $3 L_{\pi} / 8=22.85 \mu \mathrm{m}$ ) whereas a 3 fold image is recognized at the distance $z=15 \mu \mathrm{m} \simeq L_{\pi} / 4=$ $15.2 \mu \mathrm{m}$. We can also excite the waveguide in a non-symmetric way, by injecting the field in the sixth row with respect to the waveguide edge: the calculated intensity evolution is reported in Fig.7. A copy of the injected field appears at $z=56 \mu \mathrm{m}$ but the single image is mirrored with respect to the middle of the waveguide. The study of conventional waveguides having width $w$ proved that in case of an input centered at $w / 3$ from the edge of the waveguide [13], the first image should appear at $L_{\pi}=60.9 \mu \mathrm{m}$.

Since multiple images are formed, PC multimode waveguides could work as splitters (as suggested in [14],[34]). We underline how the appearance of multiple images is a consequence of the high number of supported modes (more than ten): in fact these multiple copies of the input beam have not been observed in other PC multimode devices where less than five modes could propagate [14],[15].

The mathematical conditions to observe in a $\mathrm{PC}$ waveguide a copy or a mirrored copy of the input field have been recently analyzed and a detailed procedure to find numerically the position of the images has been proposed [14]. As in our approach the starting point is the knowledge of the dispersion diagram, but such a general procedure does not lead to simple analytical formulas which are very useful for a first design. On the other hand, we verified that the formulas proposed for conventional multimode waveguides [13] give useful approximated results whenever light is well confined inside the perturbed region forming the waveguide.

\section{MODES OF PHOTONIC CRYSTAL COUPLERS}

A PC directional coupler is basically a multimode device relying on the existence and propagation of only two modes [16],[17],[35],[36],[37]. For instance if we consider two straight parallel PC waveguides separated by four unperturbed rods, the dispersion curve of the unperturbed single mode waveguide splits in two solutions: a fundamental evensymmetry mode and a second-order odd-symmetry mode [19].

It has been recently numerically observed and theoretically explained that the fundamental mode of the PC coupler can be even or odd depending on the spacing between the waveguides [18], in stark contrast with conventional couplers where the fundamental mode is always even. In the case of the square lattice PC structure we are considering, the fundamental mode is even if the spacing is composed of an even number of unperturbed rods whereas the fundamental mode is odd if the spacing is composed of an odd number of unperturbed rods [19]. The dispersion curves for a 3-rod spacing coupler (obtained from a lattice with $a=487.5 \mathrm{~nm}$ and $r=80 \mathrm{~nm}$ ) are plotted in Fig.8 and at each normalized frequency the normalized wavevector of the odd-symmetry mode is larger than the wavevector of the even-symmetry mode.

If we assume that the two single mode waveguides forming the coupler are weakly coupled, and thus the mode travelling along the isolated single mode waveguide is weakly perturbed by the presence of the other waveguide, the behavior of the coupler can be described by means of the coupled mode theory (CMT) [38]. In the framework of the CMT the field shape in each waveguide is basically that of the unperturbed waveguide but the two modes periodically exchange power along the propagation direction. The strength of the interaction between the waveguides is described by the coupling coefficient $C$

$$
C=\frac{1}{2 \beta}\left(\frac{2 \pi}{\lambda}\right)^{2} \frac{\int_{W} \delta \epsilon_{r}(x, z) E_{1}^{*}(x, z) E_{2}(x, z) d W}{\int_{W}\left|E_{1}(x, z)\right|^{2} d W},
$$

where $E_{1,2}$ are the modes of the individual waveguides (having propagation constant $\beta), \delta \epsilon_{r}(x, z)$ is the perturbation of the PC dielectric constant that introduces the defect (i.e. the reduction of the refractive index due to the removal of the high refractive index rods), and $W$ is the fundamental cell [19],[38]. It can be easily proved that the beat length $L_{\pi}$ is

$$
L_{\pi}=\frac{\pi}{2|C|},
$$

as in conventional planar couplers [26]. The field comes out from the same waveguide in which it has been injected if the coupler length is an even multiple of $L_{\pi}$ and it comes out from the other waveguide if the coupler length is an odd multiple of $L_{\pi}$.

By calculating the modes of the isolated waveguides and then the coupling coefficient (by means of Eq.(2)), we observe that $C$ is positive whenever the number of rods in the spacing is even, on the other hand $C$ is negative if the number of rods in the spacing is odd. This change of sign is due to the fact that the modes of the isolated PC waveguides have an oscillating behavior outside their "cores" (see Fig.2), and thus the sign of the numerator of Eq.(2) can be changed.

Since the sign of $C$ has a particular importance, we plot in Fig.9 the product $E_{1}{ }^{*}(x, z) E_{2}(x, z)$ (see (Eq.2)), for a 4$\operatorname{rod}(a=390 \mathrm{~nm}, r=117 \mathrm{~nm})$ and a 3-rod spacing $(a=$ $487.5 \mathrm{~nm}, r=80 \mathrm{~nm}$ ) coupler. By observing the sign of the product in the removed rod region and keeping in mind that $\delta \epsilon_{r}$ is negative (since the high refractive index rod has been removed), we can ascertain that $C>0$ for the 4-rod spacing coupler and $C<0$ for the 3 -rod spacing coupler.

The coupler is also a two-mode interference (TMI) device, supporting an even and an odd supermode (with propagation constants $\left.\beta_{\text {even,odd }}\right)$. The two modes of the 4-rod spacing and 3-rod spacing coupler are shown in Fig.10a),b) and Fig.10c),d), respectively; note that the fundamental mode is odd for the 3-rod spacing case. By comparing Eq.(1) and Eq.(3) it follows that

$$
C=\frac{\beta_{\text {even }}-\beta_{\text {odd }}}{2},
$$

which confirms that when the fundamental mode is even the coupling coefficient is positive but if the fundamental mode is odd the coupling coefficient becomes negative.

\section{ARRAYS OF COUPLED PHOTONIC CRYSTAL WAVEGUIDES}

As a coupler formed by two single-mode PC waveguides supports two modes, an array constructed by placing side by 
side $N$ PC waveguides can support up to $N$ supermodes: the PC waveguide array considered as a single guiding structure is a multimode device [39]. As an example we examine an array composed of five $\mathrm{PC}$ waveguides spaced by four unperturbed rods. The calculated dispersion relations of the five TM modes are depicted in Fig.11 and cross-sections of the modes (at $\lambda=1.55 \mu \mathrm{m}$ ) are depicted in Fig.12; noticeably these modes resemble those of an array of conventional waveguides [26].

We study by means of the TDVFEM code how the field propagates along the array for different input conditions. When the input field is the fundamental mode of the central waveguide the appearance of an input field replica can be observed. Fig. 13 shows the intensity evolution inside the array; a sharp copy of the input forms at the distance $z=45 \mu \mathrm{m}$, a double image is obtained around $z=22 \mu \mathrm{m}$ and a 4fold image at $z=15 \mu \mathrm{m}$. We then consider in Fig.14 an asymmetric input excitation: the input field is injected in the second PC waveguide from the array edge; still a copy of the input is formed at the distance $z=45 \mu \mathrm{m}$. We emphasize that the approximated relations introduced for the multimode waveguides cannot be used because the dispersion relations of the array supermodes are basically different from those of the modes of a multimode waveguide (the same conclusion is valid also in conventional waveguides [26]). In fact it is clear in Fig.11 that, if the operating wavelength is fixed, the wavevectors of the array supermodes are almost equally spaced whereas the separation of two consecutive modes of a multimode waveguide is an increasing function of the mode order (as in Fig.4).

The simulations in Fig.13 and Fig.14 clearly indicate that the device could find application as an extremely compact (shorter than $50 \mu \mathrm{m}$ ) and efficient splitter. Since the field is already confined by waveguides the transition from the array to an output single-mode waveguide does not give rise to the insertion losses observed in multimode waveguides.

It is interesting to analyse the output from the array as a function of the signal wavelength; since TDVFEM calculates the device transmission in the time domain, by propagating a suitable short pulse, the transmission spectra can be obtained from a single simulation by means of the discrete Fourier transform. Fig. 15 shows the field intensity at the outputs of a 5-waveguide array when the input is injected in the central waveguide and the wavelength $\lambda$ is varied between $1400 \mathrm{~nm}$ and $1700 \mathrm{~nm}$. Fig.15a) refers to a $22 \mu \mathrm{m}$ long array; most of the power is concentrated in the outermost waveguides for $\lambda=1550 \mathrm{~nm}$, whereas the power is equally divided among the first, second, fourth and fifth waveguide from the edge for $\lambda=1480 \mathrm{~nm}$ and the field is focused again only in the central waveguide for $\lambda=1655 \mathrm{~nm}$. Fig.15b) shows the output intensities for a $45 \mu \mathrm{m}$ long array: at $\lambda=1550 \mathrm{~nm}$ the power is concentrated in the central waveguide whereas at $\lambda=1475 \mathrm{~nm}$ the signal is equally divided among the first, second, fourth and fifth waveguide, as in a $1 \times 4$ power splitter. It is apparent that PC waveguide arrays could be used as filters and demultiplexers in order to separate signals that are more than $50 \mathrm{~nm}$ apart. Similar performance can be achieved by cascading/combining PC couplers [40],[41].

The CMT introduced for the PC directional coupler [38] can be generalized to treat the arrays [19],[20]; the only difference is that the waveguides of the array are coupled to two adjacent waveguides and not just to one as in directional couplers. The set of equations that models the propagation in an array of $N$ PC coupled waveguides is:

$$
i \frac{d A_{n}}{d z}+\beta A_{n}+C\left(A_{n+1}+A_{n-1}\right)=0, n=1, \ldots, N,
$$

where $A_{n}$ is the amplitude of the Bloch mode of the $\mathrm{n}$-th isolated PC waveguide and $\beta$ is the corresponding wavevector. Relying on the CMT the intensity evolution is explained as an exchange of power among waveguides. This energy flow is recognized in the intensity patterns depicted in Fig. 13 and Fig.14. At the edge of the array light seems to bounce back inside the array just because the field cannot be coupled to any further PC waveguide.

We observe that the light evolution along a conventional multimode waveguide resembles the diffraction experienced by a gaussian beam as long as the broadening of the input beam is much smaller than the waveguide width. Likewise if we consider an array having such a large number of waveguides that light cannot reach the array edges, discrete diffraction is clearly observed [23],[24]: in fact there are many supermodes having closely spaced propagation constants and thus the supermodes do not experience a phase difference among them sufficient to give rise to multiple images.

In Fig.16 it is depicted the intensity evolution in an array formed by eleven waveguides and having length $19 \mu \mathrm{m}$. Still we observe the formation of intensity wings along the propagation direction characterized by zero dispersion [22],[24]. For this array (4-rod spacing, $a=390 \mathrm{~nm}$ and $r=117$ $\mathrm{nm})$ the numerically calculated coupling coefficient is $C=$ $7.67 \times 10^{4} \mathrm{~m}^{-1}$ (from Eq.(2)); the array outputs have been calculated by solving the set of coupled equations (Eq.(5)) as well, and the result is shown in the right part of Fig.16. As expected, since the PC waveguides are weakly evanescently coupled, the agreement between the results from CMT and the numerical solution of Maxwell's equation is remarkably good. We have verified that if the waveguides get closer the agreement between CMT and the TDVFEM simulation gets worse: in fact the mode travelling along a waveguide is more perturbed by the presence of the nearby waveguides and the hypothesis leading to the CMT is not anymore fulfilled.

The analysis of PC couplers has shown that the sign of the coupling coefficient can be changed. In the example of Fig.16 the coupling coefficient is positive but if we considered an array with negative $C$ (which can be easily obtained by using a 3-rod spacing between the waveguides, for instance) the intensity evolution would have the same qualitative behavior. Nevertheless the change of sign of $C$ varies the sign of the phase delay experienced by the "discrete beam" travelling through the PC array. The paramount importance of the sign of $C$ has been thoroughly studied in arrays of conventional waveguides [23],[24] and it has been shown that the diffraction coefficient is given by

$$
D=-2 C d^{2} \cos \left(k_{x} d\right)
$$

where $k_{x} d$ is basically the phase difference between the field in adjacent waveguides [23]. We underline that in conventional 
arrays the sign of diffraction is controlled by varying the phase difference between the input field in the waveguides (i.e. by choosing the input tilt angle), whereas in PC arrays the sign of diffraction can be chosen by fixing the spacing between waveguides. If the spacing is composed by an even number of rods $C$ is positive and the diffraction coefficient is negative (normal diffraction); on the other hand if the spacing is formed by an odd number of rods $C$ is negative and the diffraction coefficient is positive (anomalous diffraction).

By alternating arrays with normal and anomalous diffraction it is possible to construct a structure exhibiting the desired value of average diffraction. If the average diffraction is zero, a replica of the input field is obtained at the end of the PC device. Fig. 17 shows the intensity evolution in a structure composed of a $20 \mu \mathrm{m}$ long normal diffraction array (as the one of Fig.16), followed by a $24 \mu \mathrm{m}$ long anomalous diffraction array which is followed by a $21 \mu \mathrm{m}$ long normal diffraction array. The anomalous diffraction array is composed of 3-rod spaced PC waveguides with $a=487.5 \mathrm{~nm}$ and $r=80 \mathrm{~nm}$; the geometrical parameters of the anomalous-diffraction array have been carefully chosen to avoid any abrupt transition and bend at the interface between the array sections. The input field is focused by the anomalous diffraction array at $z=32$ $\mu \mathrm{m}$ and the last section (composed by a normal diffraction array) creates a further copy of the input at $z=65 \mu \mathrm{m}$.

Intuition suggests that the interface between a normal diffraction and an anomalous diffraction PC array works like a convex lens: as the high refractive index region of the lens changes the phase front curvature of the incoming beam, in the same way the propagation in an anomalous diffraction array reshapes the curvature of the beam cancelling the phase curvature due to the propagation in the normal diffraction array.

\section{CONCLUSION}

In this paper, we studied the propagation in multimode devices constructed starting from a 2-D square lattice of dielectric rods. We analysed multimode waveguides and arrays of coupled PC waveguides and pointed out that the presence of many modes can give rise to self-imaging phenomena. We also showed how in principle the studied PC multimode devices can find application as compact $1 \times N$ splitters and demultiplexers. Moreover since diffraction can be controlled in PC waveguide arrays, our results are a significant step towards planar circuits where low-divergence optical beams can be routed along the desired paths.

Although a careful study and design must rely on TDVFEM, a comprehensive numerical tool handling time-domain Maxwell's equations, we underline the importance of the dispersion diagrams in order to explain the PC devices behavior. The simple formulas derived for conventional multimode waveguides, where the beat length between the two lowest order modes is particularly significant, provide useful indications also for the design of PC multimode waveguides whenever light is tightly confined in the waveguide.

\section{ACKNOWLEDGMENT}

The authors acknowledge financial support from Ministero dell'Istruzione, dell'Università e della Ricerca, FIRB project RBAU01XEEM and PRIN 2004 program. M. Conforti acknowledges fruitful discussions with Lucia Gastaldi.

\section{REFERENCES}

[1] J. D. Joannopoulos, R. D. Meade, and J. N. Winn, Photonic Crystals, Molding the Flow of Light. Princeton NJ: Princeton Univ. Press, 1995.

[2] W. Bogaerts, V. Wiaux, D. Taillaert, S. Beckx, B. Luyssaert, P. Bienstman, R. Baets, "Fabrication of photonic crystals in silicon-on-insulator using 248-nm deep UV lithography," IEEE J. Sel. Topics Quantum Electron., vol. 8, pp. 928-934, Aug. 2002.

[3] T. F. Krauss, and R. M. De La Rue, "Photonic crystals in the optical regime-Past, present and future," Prog. Quantum. Electron., vol. 23, pp. $51-96,1999$.

[4] S. G. Johnson, P. R. Villeneuve, S. Fan, and J. D. Joannopoulos, "Linear waveguides in photonic-crystal slabs," Phys. Rev. B, vol. 62, pp. 82128222, Sep. 2000.

[5] A. Martinez, J. Garcia, G. Sanchez, and J. Marti, "Planar photonic crystal structure with inherently single-mode waveguides," J. Opt. Soc. Am. B, vol. 20, pp. 2131-2136, Nov. 2003.

[6] S. G. Johnson, P. Bienstman, M. A. Skorobogatiy, M. Ibanescu, E. Lidorikis, and J. D. Joannopoulos, "Adiabatic theorem and continuous coupled-mode theory for efficient taper transitions in photonic crystals," Phys. Rev. E, 066608, 2002.

[7] M. Lončar, T. Doll, J. Vučković, A. Scherer, "Design and fabrication of silicon photonic crystal optical waveguides," J. Lightwave Technol., vol. 18 , pp. 1402-1411, Oct. 2000.

[8] H. Benisty, D. Labilloy, C. Weisbuch, C. J. M. Smith, T. F. Krauss, D. Cassagne, A. Béraud, and C. Jouanin, "Radiation losses of waveguidebased two-dimensional photonic crystals: positive role of the substrate," Appl. Phys. Lett., vol. 76, pp. 532-534, 2000.

[9] L. B. Soldano, F. B. Veerman, M. K. Smit, B. H. Verbeek, A. H. Dubost, and E. C. M. Pennings, "Planar monomode optical couplers based on multimode interference effects," J. Lightwave Technol., vol. 10, pp. 18431850, Dec. 1992

[10] D. S. Levy, K. Hyun Park, R. Scarmozzino, R. M. Osgood, C. Dries, P. Studenkov, and S. Forrest, "Fabrication of ultracompact 3-dB $2 \times 2$ MMI power splitters," IEEE Photon. Technol. Lett., vol. 11, pp. 10091011, Aug. 1999.

[11] R. Ulrich, and G. Ankele, "Self-imaging in homogeneous planar optical waveguides," Appl. Phys. Lett., vol. 27, pp. 337-339, 1975.

[12] M. Bachmann, P. A. Besse, and H. Melchior, "General self-imaging: properties in $N \times N$ multimode interference couplers including phase relations," Appl. Opt., vol. 33, pp. 3905-3911, Jun. 1994.

[13] L. B. Soldano, and E. C. M. Pennings, "Optical multi-mode interference devices based on self-imaging: principles and applications," J. Lightwave Technol., vol. 13, pp. 615-627, Apr. 1995.

[14] H. J. Kim, I. Park, B. H. O, S. G. Park, E. H. Lee, and S. G. Lee, "Self-imaging phenomena in multi-mode photonic crystal line-defect waveguides: application to wavelength de-multiplexing ," Optics Express, vol. 12 , pp. 5625-5633, Nov. 2004.

[15] I. Park, H. S. Lee, H. J. Kim, K. M. Moon, S. G. Lee, B. H. O, S. G. Park, and E. H. Lee, "Photonic crystal power-splitter based on directional coupling ," Optics Express, vol. 12, pp. 3599-3604, Jul. 2004.

[16] S. Boscolo, M. Midrio, and C. G. Someda, "Coupling and decoupling of electromagnetic waves in parallel 2-D photonic crystal waveguides," IEEE J. Quantum Electron., vol. 38, pp. 47-53, Jan. 2002.

[17] A. Martinez, F. Cuesta, and J. Marti, "Ultrashort 2-D photonic crystal directional couplers," IEEE Photon. Technol. Lett., vol. 15, pp. 694-696, May 2003.

[18] C. M. de Sterke, L. C. Botten, A. A. Asatryan, T. P. White, and R. C. McPhedran, "Modes of coupled photonic crystal waveguides," Opt. Lett., vol. 29, pp. 1384-1386, Jun. 2004.

[19] A. Locatelli, M. Conforti, D. Modotto, C. De Angelis, "Diffraction engineering in arrays of photonic crystal waveguides," Opt. Lett., vol. 30, pp. 2894-2896, Nov. 2005.

[20] A. Locatelli, M. Conforti, D. Modotto, C. De Angelis, "Discrete negative refraction in photonic crystal waveguide arrays," accepted for publication by $O$ Pt. Lett.

[21] D. Christodoulides and R. Joseph, "Discrete self-focusing in nonlinear arrays of coupled waveguides," Opt. Lett., vol. 13, pp. 794-796, Sep. 1988. 
[22] H. S. Eisenberg, Y. Silberberg, R. Morandotti, A. R. Boyd, and J. S. Aitchison, "Discrete spatial optical solitons in waveguide arrays," Phys. Rev. Lett., vol. 81, pp. 3383-3386, 1998.

[23] H. S. Eisenberg, Y. Silberberg, R. Morandotti, and J. S. Aitchison, "Diffraction management," Phys. Rev. Lett., vol. 85, pp. 1863-1866, 2000.

[24] T. Pertsch, T. Zentgraf, U. Peschel, A. Bräuer, and F. Lederer, "Anomalous refraction and diffraction in discrete optical systems," Phys. Rev. Lett., vol. 88, 093901, 2002.

[25] S. G. Johnson, and J. D. Joannopoulos, "Block-iterative frequencydomain methods for Maxwell's equations in a planewave basis," Optics Express, vol. 8, pp. 173-190, Jan. 2001

[26] A. Yariv, Optical Electronics in Modern Communications. Oxford Univ. Press, 1997.

[27] D. White, G. Rodrigue, "Improved vector FEM solutions of Maxwell's equations using grid pre-conditioning," Int. J. Numer. Meth. Engng., vol. 40, pp. 3815-3837, 1997.

[28] P. Ciarlet, J. Zou, "Fully discrete finite element approaches for timedependent Maxwell's equations," Numer. Math., vol. 82, pp. 193-219, 1999.

[29] G. Rodrigue, D. White, "A vector finite element time-domain method for solving Maxwell's equations on unstructured hexahedral grids," SIAM J. Sci. Comput., vol. 23, pp. 683-706, 2001.

[30] J. Lee, R. Lee, A. Cangellaris, "Time-Domain Finite-Element Methods", IEEE Trans. Antennas Propagat., vol. 45, pp. 430-442, Mar. 1997.

[31] J. C. Nedelec, "Mixed finite elements in $\mathbf{R}^{3}$ ", Numer. Math., vol. 35, pp. 315-341, 1980.

[32] D. Boffi, M. Conforti, L. Gastaldi, "Modified edge finite elements for photonic crystals", accepted for publication by Numer. Math.

[33] D. Boffi, P. Fernandes, L. Gastaldi, I. Perugia, "Computational models of electromagnetic resonators: analysis of edge element approximation", SIAM J. Numer. Anal., vol. 36, pp. 1264-1290, 1999.

[34] T. Liu, A. R. Zakharian, M. Fallahi, J. V. Moloney, and M. Mansuripur, "Multimode interference-based photonic crystal waveguide power splitter," J. Lightwave Technol., vol. 22, pp. 2842-2846, Dec. 2004.

[35] S. Kuchinsky, V. Y. Golyatin, A. Y. Kutikov, T. P. Pearsall, D. Nedeljkovic, "Coupling between photonic crystal waveguides," IEEE J. Quantum. Electron., vol. 38, pp. 1349-1352, Oct. 2002.

[36] M. Thorhauge, L. H. Frandsen, and P. I. Borel, "Efficient photonic crystal directional couplers," Opt. Lett., vol. 28, pp. 1525-1527, Sep. 2003.

[37] G. Chen, and J. U. Kang, "Waveguide mode converter based on twodimensional photonic crystals," Opt. Lett., vol. 30, pp. 1656-1658, Jul. 2005.

[38] T. Kamalakis, and T. Sphicopoulos, "Numerical study of the implications of size nonuniformities in the performance of photonic crystal couplers using coupled mode theory," IEEE J. Quantum Electron., vol. 41, pp. 863-871, Jun. 2005.

[39] L. C. Botten, R. A. Hansen, and C. M. de Sterke, "Supermodes in multiple coupled photonic crystal waveguides," Optics Express, vol. 14, pp. 387-396, Jan. 2006.

[40] M. Koshiba, "Wavelength division multiplexing and demultiplexing with photonic crystal waveguide couplers," J. Lightwave Technol., vol. 19, pp. 1970-1975, Dec. 2001

[41] F. S. S. Chien, Y. J. Hsu, W. F. Hsieh, and S. C. Cheng, "Dual wavelength demultiplexing by coupling and decoupling of photonic crystal waveguides ," Optics Express, vol. 12, pp. 1119-1125, Mar. 2004.

Daniele Modotto received the Laurea degree (cum laude) in electronic engineering and the Ph.D. degree in telecommunications from the University of Padova, Padova, Italy, in 1996 and 2000, respectively. From 2000 to 2001, he was with the Department of Electronics and Electrical Engineering, University of Glasgow, UK, where he was involved in nonlinear wavelength conversion modeling. Since November 2001, he has been working at the University of Brescia, Brescia, Italy. His research interests are nonlinear optics and passive waveguide components for telecommunications.
Matteo Conforti was born in Brescia, Italy, in 1978. He studied at the University of Brescia, where he received the Laurea degree in electronic engineering in 2003. He is working toward the Ph.D. degree at the Department of Electronics of the University of Brescia. His main research interests include nonlinear optics and numerical methods for electromagnetism.

Andrea Locatelli was born in Seriate, Bergamo, Italy, in 1977. He received the Laurea degree (cum laude) in electronic engineering and the Ph.D. degree in information engineering from the University of Brescia, Brescia, Italy, in 2001 and 2004, respectively. His main research interests include nonlinear optics and numerical modelling of waveguide components for telecommunications.

Costantino De Angelis was born in Padova, Italy, in 1964. He received the Laurea degree (cum laude) in electronic engineering and the Ph.D. degree in telecommunications from the University of Padova, Padova, Italy, in 1989 and 1993 respectively. From 1993 to 1994, he was with the Department of Mathematics and Statistics of the University of New Mexico at Albuquerque, NM. From 1995 to 1997, he was Assistant Professor at the Department of Electronics and Informatics of the University of Padova, Italy. From 1997 to 1998, he was with IRCOM ("Institut de Recherche en Communications Optiques et Microondes") at the University of Limoges, France. Since 1998, he is professor of electromagnetic fields at the University of Brescia, Brescia, Italy. His fields of technical interest are optical communications, soliton propagation, and photonics. He has authored or coauthored about 150 among papers and conference contributions. 


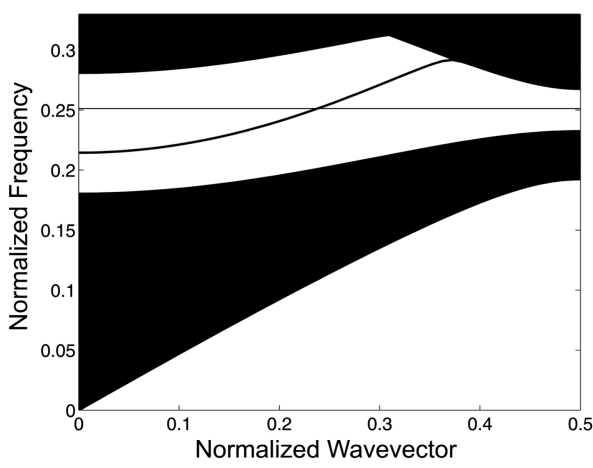

Fig. 1. Projected band structure for the waveguide obtained by removing a row of dielectric rods from a square lattice of semiconductor rods ( $n=3.34$ ) embedded in oxide $(n=1.5)$. The lattice constant is $a=390 \mathrm{~nm}$ and the rod radius is $r=117 \mathrm{~nm}$.

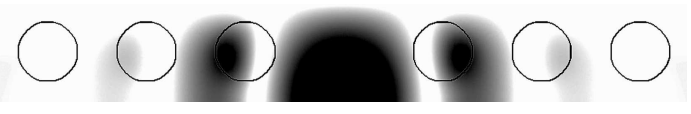

Fig. 2. Waveguide 2-D mode shape in the center of the cell; the circles indicate the position of the rods.

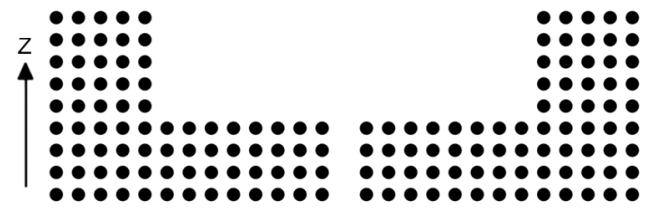

Fig. 3. Sketch of the multimode device: the large waveguide is obtained by removing seventeen rows of rods. The input single row and single-mode waveguide is used to inject the light.

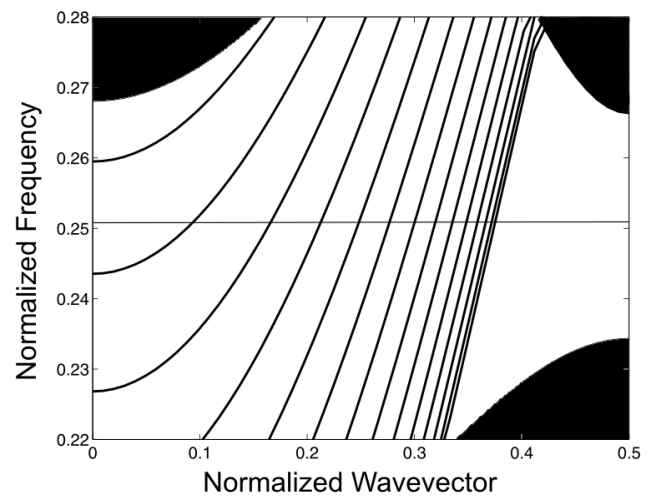

Fig. 4. Projected band structure of the 17-rod wide waveguide. At the normalized frequency $a / \lambda=0.2516(\lambda=1550 \mathrm{~nm})$ there are thirteen guided modes.

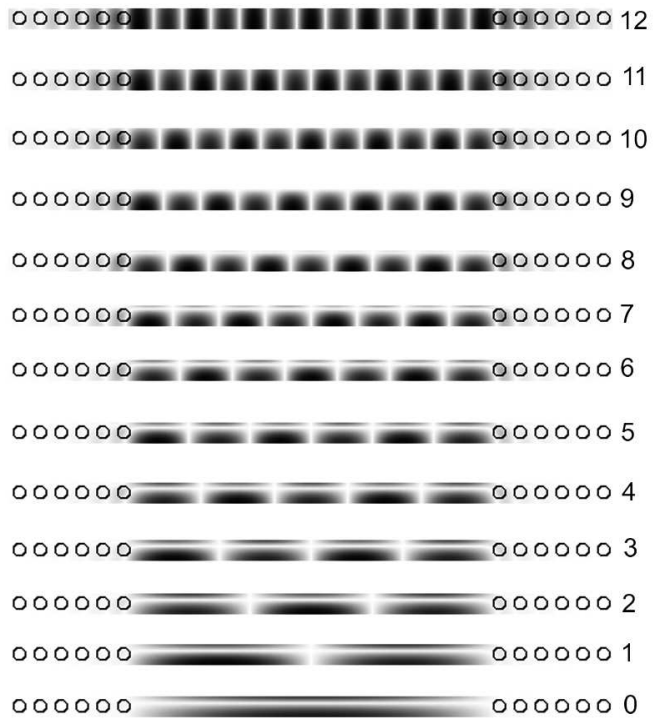

Fig. 5. The thirteen 2-D supermodes of the 17-rod wide multimode waveguide (at $\lambda=1550 \mathrm{~nm}$ ). The dielectric rods are indicated as black circles.

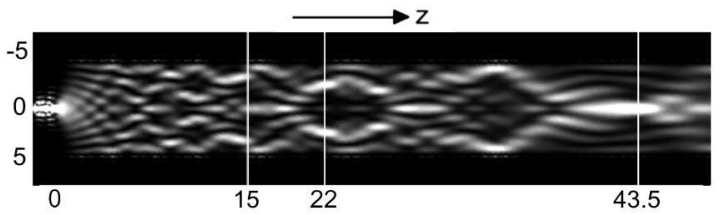

Fig. 6. Intensity evolution along the 17-rod wide device for a symmetric input (simulated by TDVFEM); white refers to the highest intensity. The first copy of the input is formed at $z=43.5 \mu \mathrm{m}$, a 2 -fold image is obtained at $z=22 \mu \mathrm{m}$ and a 3 -fold image at $z=15 \mu \mathrm{m}$.

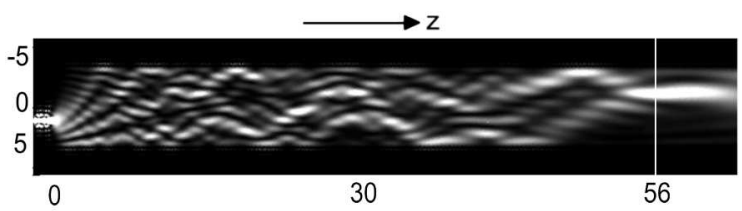

Fig. 7. Intensity evolution along the 17-rod wide device for an asymmetric input: the field is injected in the 6-th rod from the edge (simulated by TDVFEM). A mirrored copy of the input is formed at $z=56 \mu \mathrm{m}$.

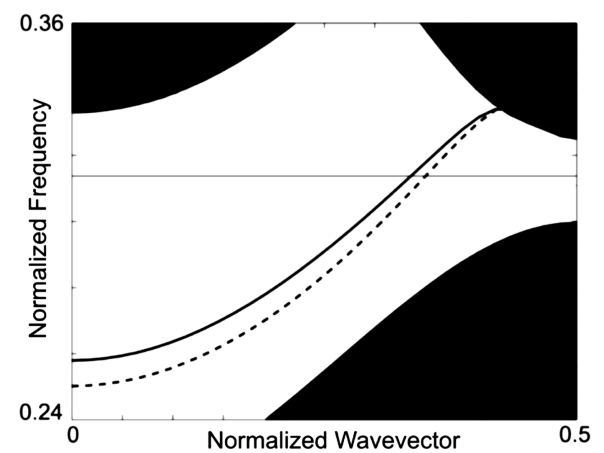

Fig. 8. Dispersion relations for the supermodes of the 3-rod spacing coupler ( $a=487.5 \mathrm{~nm}, r=80 \mathrm{~nm}$ ); odd supermode (dashed line) and even supermode (solid line) 

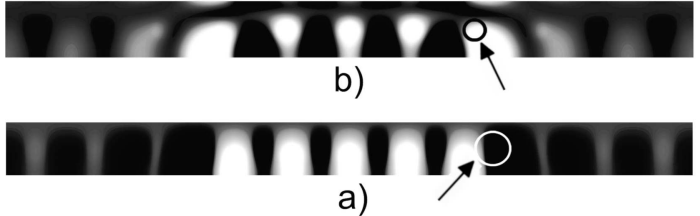

a)

Fig. 9. Product $E_{1}^{*} E_{2}$ between the modal fields of adjacent individual waveguides (as used in the formula for $C$ ). a) 4-rod spacing coupler, the white circle delimits the defect area (the adjacent waveguide removed rod). b) 3-rod spacing coupler, the black circle delimits the defect area. Colormap: black for negative values, white for positive values.

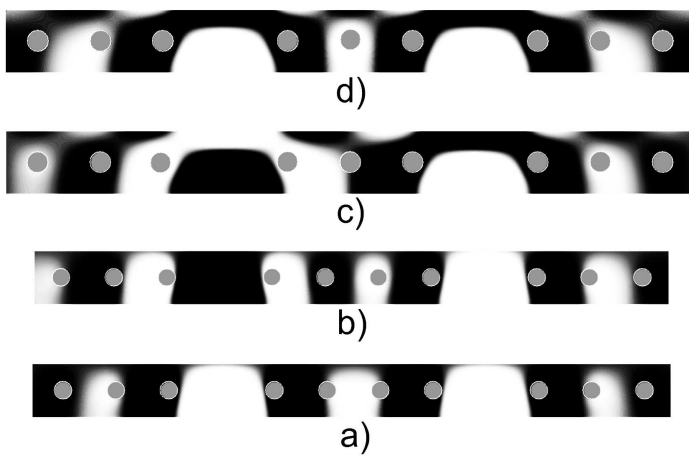

Fig. 10. a) Fundamental and b) second-order supermode of the 4-rod spacing coupler and c) fundamental and d) second-order supermode of the 3-rod spacing coupler. The superimposed grey circles show the position of the rods. Colormap: white for positive values of the field and black for negative values.

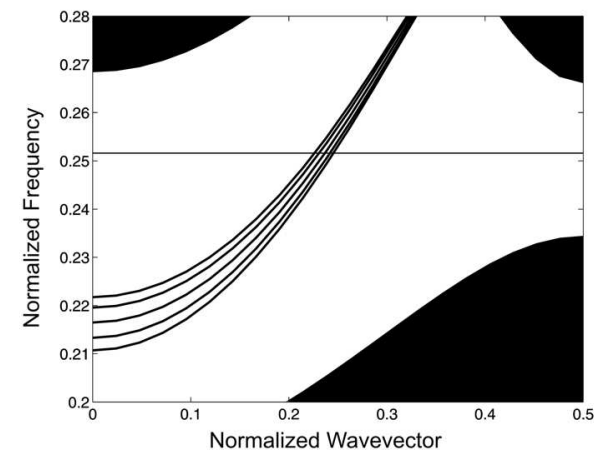

Fig. 11. Projected band structure for the supermodes of an array formed by five waveguides spaced by four rods $(a=390 \mathrm{~nm}, r=117 \mathrm{~nm})$.

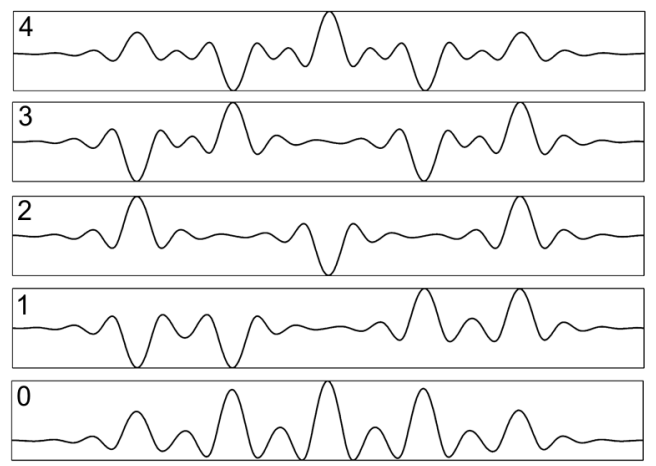

Fig. 12. Cross sections of the supermodes (at $\lambda=1550 \mathrm{~nm}$ ) of an array formed by five waveguides spaced by four rods $(a=390 \mathrm{~nm}, r=117 \mathrm{~nm})$.

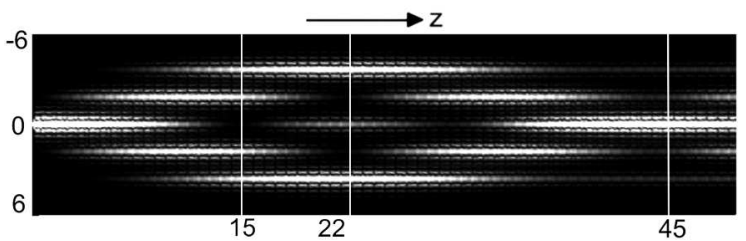

Fig. 13. Intensity evolution in the 5-waveguide array for a symmetric input (simulated by TDVFEM). The first image is formed at $z=45 \mu \mathrm{m}$. A 2-fold image and a 4-fold image are observed at $z=22 \mu \mathrm{m}$ and $z=15 \mu \mathrm{m}$, respectively.

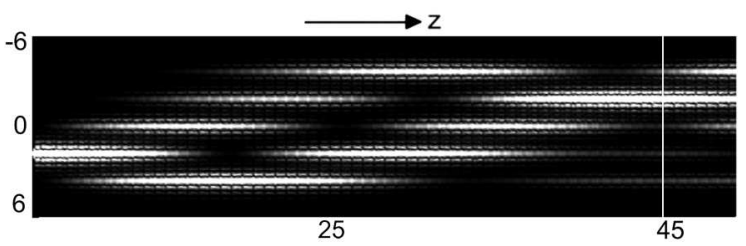

Fig. 14. Intensity evolution in the 5-waveguide array, asymmetric input: the field is injected in the second waveguide from the edge. A mirrored copy forms at $z=45 \mu \mathrm{m}$.

b)

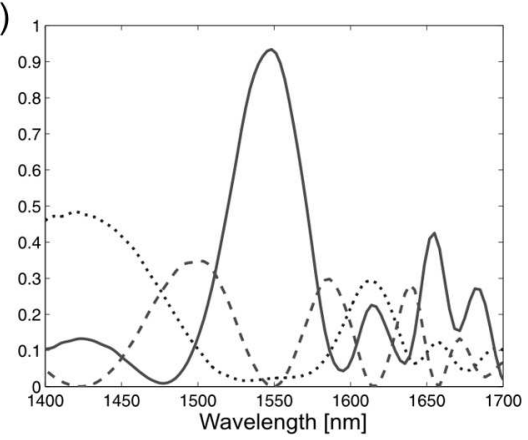

a)

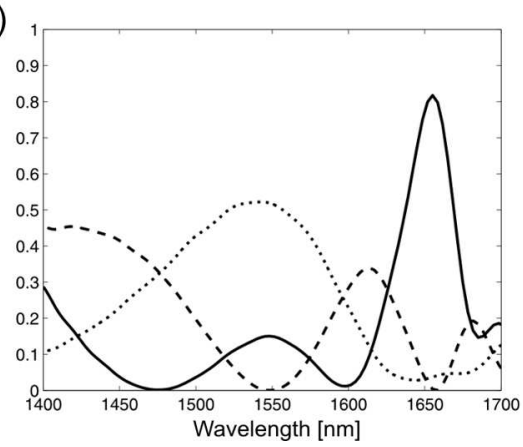

Fig. 15. Outputs as a function of the input wavelength when the field is injected in the central waveguide (simulated by means of TDVFEM). a) 22 $\mu \mathrm{m}$ long, 5-waveguide array (4-rod spacing); b) $45 \mu \mathrm{m}$ long, 5-waveguide array (4-rod spacing). Solid line: output from the central waveguide; dashed line: output from the second and fourth waveguide (from one edge); dotted line: output from the first and fifth waveguide. 


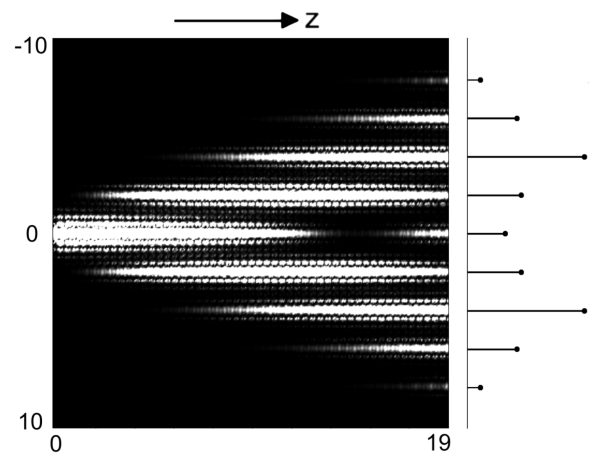

Fig. 16. TDVFEM simulation of the 11-waveguide normal-diffraction array ( $a=390 \mathrm{~nm}, r=117 \mathrm{~nm}$, 4-rod spacing) and comparison with the outputs calculated by means of the CMT with $C=7.67 \times 10^{4} \mathrm{~m}^{-1}$ and $\beta=$ $3.78 \times 10^{6} \mathrm{~m}^{-1}$ (on the right).

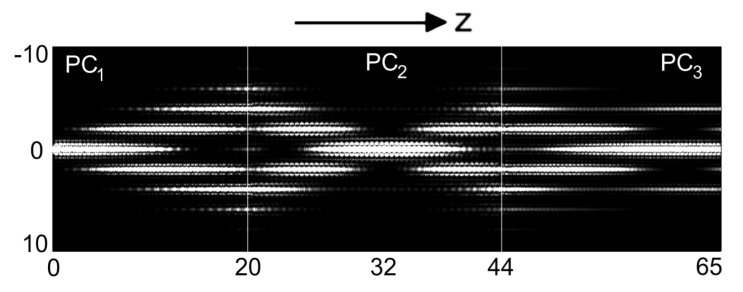

Fig. 17. Intensity evolution along sections of $\mathrm{PC}$ waveguide arrays. $\mathrm{PC}_{1}$ : normal-diffraction array, length $20 \mu \mathrm{m}$. $\mathrm{PC}_{2}$ : anomalous-diffraction array, length $24 \mu \mathrm{m} . \mathrm{PC}_{3}$ : normal-diffraction array, length $21 \mu \mathrm{m}$. A first copy of the input image is formed at $z=32 \mu \mathrm{m}$. 